\title{
Parâmetros da fase de vida livre de Rhipicephalus sanguineus (Latreille, 1806) (Acari: Ixodidae): adaptado ao clima subtropical
}

\author{
Parameters of free-living phase of Rhipicephalus sanguineus \\ (Latreille, 1806) (Acari: Ixodidae): adapted to the subtropical climate
}

\author{
Cíntia Lidiane Guidotti Aguiar ${ }^{1 *}$, Diego Moscarelli Pinto², Felipe Geraldo Pappen ${ }^{3}$, \\ Nilton Azevedo da Cunha Filho', Tânia Regina Bettin dos Santos ${ }^{4}$, Nara Amélia da Rosa Farias ${ }^{5}$
}

| | | | | | | | | | | | | | | | | | | | | | | | | | | | | | | | | | | | | | | | | | | | | | | | | | | | | | | | | | | | | | | | | | | | | | | | | | | | | | | | | | | | | | | | | | | | | | | | | | | | | | | | | | | | | | | | | | | | | | | | | | | | | | | | | | | | | | | | | | | | | | | | | | | | | | | | | | | | | | | | | | | | | | | | | | | | | | | | | | | | | | | | | | | | | | | | | | | | | | | | | | | | | | |

RESUMO: Rhipicephalus sanguineus é considerada a espécie de ixodídeo mais prevalente no mundo e foi introduzida no meio urbano pelo cão doméstico, seu principal hospedeiro. $\mathrm{O}$ presente estudo teve por objetivo conhecer o desempenho biológico da fase de vida livre de $R$. sanguineus, adaptado à regiáo Sul do Rio Grande do Sul, por meio da estimativa de parâmetros biológicos, sob condiçôes controladas de temperatura $\left(27 \pm 1^{\circ} \mathrm{C}\right)$ e umidade relativa $(>70,0 \%)$. Foram coletadas teleóginas pré e pós-desprendimento dos cães hospedeiros, sendo cada grupo experimental constituído de 200 teleóginas. Verificou-se que a massa média de cada teleógina foi de $170 \mathrm{mg}$, o período de pré-postura de quatro dias, a massa total de ovos de $100 \mathrm{mg}$, a massa da quenógina de $30 \mathrm{mg}$ e a longevidade larval de 31 dias, com índices de eficácia reprodutiva e nutricional de 61,0 e 74,0\%, respectivamente. Quanto à dinâmica de postura, 98,5\% foram ovipostos na primeira semana de incubaçáo e a eclodibilidade foi de $94,5 \%$. A análise dos parâmetros biológicos de $R$. sanguineus revelou que não houve diferença estatística $(\mathrm{p}>0,05)$ entre as fêmeas coletadas diretamente do corpo do hospedeiro e aquelas provenientes do ambiente. Os dados biológicos observados em $R$. sanguineus adaptados às condições climáticas do Sul do Rio Grande do Sul são similares aos de outras regiōes do Brasil. Além disso, as coletas de $R$. sanguineus para estudos epidemiológicos podem ser realizadas tanto do ambiente quanto do corpo do hospedeiro, devido à similaridade que ambos os grupos apresentam em seus parâmetros biológicos.

PALAVRAS-CHAVE: biologia; carrapato; cão.

\begin{abstract}
Rhipicephalus sanguineus is considered the most widespread ixodid species in the world. It was introduced in the urban area by the domestic dog, which is its primary host. The present study had as its aim to know the biological performance of the freeliving phase of $R$. sanguineus, adapted to the Southern of Rio Grande do Sul, through estimation of biological parameters under controlled temperature conditions $\left(27 \pm 1^{\circ} \mathrm{C}\right)$ and relative humidity $(>70.0 \%)$. Engorged females ticks were collected from the environment and attached to the body of dogs and each group consisted of 200 ticks. The obtained results demonstrated that the mean mass of each engorged female was $170 \mathrm{mg}$, the pre-oviposition period was four days, the total eggs mass weight was of $100 \mathrm{mg}$, the mass of female tick after laying was $30 \mathrm{mg}$, larval longevity has 31 days, and reproductive and nutrition efficacy index corresponded to 61 and $74 \%$ respectively. Regarding posture dynamic, $98.5 \%$ of eggs were positioned in the first week of incubation and the larval hatching rate was $94.5 \%$. The biological parameter analysis of $R$. sanguineus showed that there was no statistical difference $(\mathrm{p}>0.05)$ between females collected directly from the host body and those from the environment. Biological data seen in $R$. sanguineus adapted to climate conditions of South Rio Grande do Sul are similar to other Brazilian areas. Furthermore, $R$. sanguineus collects for epidemiological studies can be carried out both from dogs and from the environment, due to the similarity that both groups present in their biological parameters.
\end{abstract}

KEYWORDS: biology; tick; dog.

\footnotetext{
'Faculdade de Veterinária (FV), Programa de Pós-Graduação em Veterinária, Universidade Federal de Pelotas (UFPel) - Pelotas (RS), Brasil.

¿'Universidade Federal do Pampa (UNIPAMPA) - Uruguaiana (RS), Brasil.

${ }^{3}$ Instituto Federal Catarinense (IFC) - Concórdia (SC), Brasil.

${ }^{4}$ Departamento de Medicina Veterinária Preventiva, UFPel - Pelotas (RS), Brasil.

${ }^{5}$ Departamento de Microbiologia e Parasitologia, UFPel - Pelotas (RS), Brasil.

*Autor correspondente: cintia-aguiar@ig.com.br

Recebido em: 23/04/2012. Aceito em: 15/08/2013
} 


\section{INTRODUÇÃO}

Rhipicephalus sanguineus é originário da África, sendo conhecido como "carrapato vermelho do cáo" e está amplamente distribuído, sendo, provavelmente, a espécie de ixodídeo mais prevalente no mundo (Pegram et al., 1987). Apesar de sua origem afrotropical, sua distribuição quase cosmopolita deve-se aos movimentos migratórios do homem, levando consigo o cáo doméstico, seu principal hospedeiro (WALKer et al., 2000).

Devido ao fato de exercer hematofagia, esse carrapato é o principal vetor natural e de importância reconhecida para Babesia canis e Ehrlichia canis, para cães, e Rickettsia conorii, para humanos (Labruna; Pereira, 2001; Labruna, 2004). Estudos recentes revelaram um novo potencial para $R$. sanguineus, podendo esse ixodídeo estar envolvido na epidemiologia da leishmaniose visceral canina, além da possibilidade de transmitir $R$. rickettsii, agente etiológico da febre maculosa brasileira (Labruna et al., 2008; Cunha et al., 2009; Dantas-Torres et al., 2010).

Observaçóes realizadas a partir de dados morfológicos e moleculares demonstraram haver diferenças significativas entre as populaçóes de $R$. sanguineus do Brasil e da Argentina, sugerindo a existência de dois grupos distintos no Sul da América (Oliveira et al., 2005; Szabó et al., 2005). No entanto, novas investigaçóes acerca da variabilidade genotípica de $R$. sanguineus estáo sendo realizadas, pois BurLini $e t$ al. (2010) indicaram a existência de uma relativa diferença intraespecífica entre as populações desse carrapato, as quais são provenientes de várias regiôes do Brasil.

Mudanças nas condições climáticas poderão ter um impacto indireto na dinâmica populacional de tal ixodídeo, contribuindo para uma maior abundância de carrapatos e, assim, aumentando o tempo de circulação e transmissão de patógenos (Gray et al., 2009).

No Brasil, trabalhos sobre a biologia em condiçôes controladas de temperatura e umidade foram realizados por Coelho (1993), Bechara et al. (1995), Sartor et al. (1996) e Bellato; Daemon (1997), estudando populaçóes adaptadas a condições de clima tropical, fato que diminui as chances de um controle efetivo das infestaçôes por esse parasito.

Nos últimos anos, tem ocorrido um aumento considerável da infestação por $R$. sanguineus em câes das áreas urbanas da regiáo Sul do Rio Grande do Sul. O problema tem se agravado ano após ano, com queixas da populaçáo da invasão de residências pelo carrapato ou até mesmo casos de parasitismo em humanos (sobretudo crianças), o que representa um risco à saúde. O desconhecimento sobre o comportamento do ixodídeo na regiāo e seus parâmetros biológicos dificultam ainda mais as tentativas de controle feitas pelos órgãos responsáveis, uma vez que, até o momento, não foram realizados trabalhos científicos sobre o tema no Estado do Rio Grande do Sul, Brasil.
O presente estudo teve por objetivos: conhecer o desempenho biológico, em condiçóes laboratoriais, da fase de vida livre de $R$. sanguineus na regiáo Sul do Rio Grande do Sul e comparar os dados obtidos das teleóginas coletadas diretamente do corpo dos cáes hospedeiros com aquelas do ambiente (desprendidas naturalmente).

\section{MATERIAL E MÉTODOS}

\section{Unidades experimentais}

O experimento foi realizado por meio da análise de 200 teleóginas coletadas diretamente dos cães e outras 200 do ambiente. Cada indivíduo constituiu uma unidade experimental.

\section{Coleta do material}

As coletas de carrapatos ocorreram em cães e nas residências localizadas na periferia da cidade, de 2007 a 2010. Em tais bairros e vilas, o parasitismo mostrou-se mais grave, segundo informaçóes da Secretaria Municipal de Saúde de Pelotas.

Os carrapatos foram coletados manualmente de seus hospedeiros, desde que estivessem ingurgitados e com um comprimento mínimo de $0,8 \mathrm{~cm}$. Foram coletados carrapatos do ambiente (muros, paredes, areia, entre outros) e aqueles com um grau de ingurgitamento indicando que ainda não haviam iniciado a postura. Após a coleta, as unidades experimentais foram acondicionadas em recipiente fechado e levadas ao Laboratório de Parasitologia do Instituto de Biologia da Universidade Federal de Pelotas. As fêmeas ingurgitadas foram identificadas morfologicamente a partir das chaves de identificação e descrição da espécie, segundo WALKER et al. (2000).

\section{Processamento}

Inicialmente, as teleóginas foram lavadas em água corrente, secas em papel absorvente e pesadas individualmente em balança analítica. Em seguida, foram acondicionadas em placas de Petri individuais, devidamente identificadas e levadas à estufa do tipo $\mathrm{BOD}$, temperatura de $27 \pm 1^{\circ} \mathrm{C}$, e umidade relativa superior a 70\% (Bellato; DAEMon, 1997).

\section{Análise dos parâmetros biológicos}

Foram realizadas observações diárias para a obtenção de dados relativos à evolução biológica de cada unidade experimental. Os parâmetros biológicos analisados foram:

- massa da teleógina (mg) - massa da fêmea de carrapato antes de iniciar a postura; 
- $\quad$ período de pré-postura (dias) - compreendido entre a coleta da fêmea ingurgitada e o início da oviposição;

- dinâmica de postura (\%) - determinada pela coleta e pesagem da massa de ovos produzida a cada sete dias de oviposição;

- massa de ovos produzida (mg) - massa total de ovos realizada desde o início até o final da postura;

- massa da quenógina (mg) - massa da fêmea ao final de sua postura;

- $\quad$ eclodibilidade (\%) - percentual estimado de larvas eclodidas em relação ao total de ovos incubados, sendo avaliado após o final da eclosão;

- longevidade larval (dias) - período decorrido desde o início da eclosão até a morte da última larva;

- Índice de Eficiência Reprodutiva (IER) e Índice Nutricional (IN), calculados de acordo com as equaçóes abaixo (BENETT, 1974):

IER = (massa total de ovos/massa inicial da teleógina $)$ x 100

IN $=$ [massa total de ovos/(massa inicial da teleógina - massa final da teleógina)] x 100

\section{Análise estatística}

Foram utilizadas análise de variância - ANOVA $(\mathrm{p} \leq 0,05) \mathrm{e}$ o teste de Tukey para comparar os parâmetros biológicos de cada grupo (cão e ambiente), por meio do programa Statistix 9.0 (Tallahasse, 2008).

\section{RESULTADOS}

As teleóginas obtidas do ambiente foram encontradas escondidas na casa de seu hospedeiro, em tocas, frestas de paredes e postes, caminhando em muros ou até mesmo enterradas na areia a uma profundidade de até $10 \mathrm{~cm}$, o que comprova o hábito essencialmente nidícola de tal carrapato.

No decorrer das visitas, notou-se um aumento no número de residências que apresentavam altas infestaçóes, nas quais pessoas conviviam com carrapatos que invadiam suas casas. Nas habitaçóes, as fêmeas em vários graus de ingurgitamento estavam escondidas nas paredes próximas ao teto.

Foram encontradas poucas teleóginas parasitando os cães, mesmo em ambientes altamente infestados, indicando que o ingurgitamento ocorre, sobretudo, à noite. Apesar de serem raros os cães que apresentam altas infestaçôes, alguns canídeos estavam altamente parasitados, sugerindo que alguns deles sejam mais suscetíveis a esse carrapato. Um cão sem raça definida (SRD), com cerca de cinco anos, foi encontrado altamente infestado em sucessivas visitas. Os dados referentes aos parâmetros biológicos encontram-se na Tabela 1 .

De acordo com os resultados, a massa média das teleóginas foi igual em ambos os grupos (170 mg), sendo observadas as mesmas semelhanças quanto a outros parâmetros, tais como: massa total de ovos (100 mg), massa das quenóginas (30 mg) e período de pré-postura (quatro dias).

Em relação à dinâmica de postura, 99,0 e 98,0\% dos ovos foram postos durante a primeira semana, respectivamente, pelas fêmeas coletadas do hospedeiro e do ambiente. As médias dos índices de eclodibilidade variaram entre 94,0 e $95,0 \%$.

Quanto à longevidade larval dos grupos de teleóginas provenientes do ambiente e aquelas coletadas diretamente do corpo do hospedeiro, demonstrou-se semelhança, sendo 31 dias o período constatado para esta variável em ambos os grupos.

Quanto ao IER e IN, os resultados foram de 61,0 e $74,0 \%$, respectivamente, para ambas as origens.

A análise estatística revelou que não existem diferenças significativas ( $\mathrm{p}>0,05)$ entre os parâmetros biológicos das fêmeas de $R$. sanguineus coletadas diretamente do corpo do hospedeiro e os de teleóginas do ambiente (desprendidas naturalmente).

Tabela 1. Parâmetros biológicos de $R$. sanguineus naturalmente desprendidas e coletadas do corpo do hospedeiro, com dados referentes à amplitude, à média e ao desvio padrão.

\begin{tabular}{|c|c|c|c|c|c|c|}
\hline \multirow{3}{*}{ Parâmetros } & \multicolumn{6}{|c|}{ Origem da teleógina } \\
\hline & \multicolumn{3}{|c|}{ Ambiente } & \multicolumn{3}{|c|}{ Corpo do cão } \\
\hline & Amplitude & Média & DP & Amplitude & Média & DP \\
\hline Massa da teleógina (mg) & $90-250$ & $170^{a}$ & 30 & $70-360$ & $170^{a}$ & 60 \\
\hline Período de pré-postura (dias) & $1-7$ & $3,9^{a}$ & 0,7 & $2-7$ & $4,1^{\mathrm{a}}$ & 0,6 \\
\hline Massa total de ovos (mg) & $40-190$ & $100^{a}$ & 20 & $2-240$ & $100^{a}$ & 40 \\
\hline Ovos postos na primeira semana (\%) & $81-99$ & $99^{a}$ & 1,2 & $80-99$ & $98^{a}$ & 0,6 \\
\hline Massa da quenógina (mg) & $10-60$ & $30^{a}$ & 7 & $1-70$ & $30^{a}$ & 10 \\
\hline Eclodibilidade (\%) & $90-99$ & $94^{\mathrm{a}}$ & 2,8 & $90-99$ & $95^{\mathrm{a}}$ & 2,8 \\
\hline Longevidade larval (dias) & $31-32$ & $31^{a}$ & 0,4 & $29-34$ & $31^{\mathrm{a}}$ & 2,2 \\
\hline Índice de Eficácia Reprodutiva (\%) & $27-90$ & $61^{a}$ & 7,2 & $3,0-82$ & $61^{\mathrm{a}}$ & 10,1 \\
\hline Índice Nutricional (\%) & $34-100$ & $74^{a}$ & 9,7 & $3-100$ & $74^{\mathrm{a}}$ & 12,9 \\
\hline
\end{tabular}

a médias seguidas da mesma letra na linha não diferem estatisticamente entre si ( $p>0,05)$. 


\section{DISCUSSÃO}

Durante as coletas, comprovou-se o hábito essencialmente nidícola do carrapato em estudo, o que deve ser levado em conta em programas de controle, os quais devem ser direcionados ao ambiente, uma vez que uma pequena parcela da população deste carrapato se encontra sobre o cáo hospedeiro (Labruna, 2004).

Para que o tratamento e controle das infestaçóes por $R$. sanguineus tenham efeito sobre essas populações, as aplicaçóes de acaricida devem ser realizadas em locais onde o carrapato esteja escondido (tocas e frestas). No caso de terrenos arenosos, é necessária a remoção da camada superficial de areia para que as teleóginas enterradas fiquem expostas ao produto e, assim, segundo Labruna; Pereira (2001), sejam atingidos os $95,0 \%$ dos carrapatos que estão em tal ambiente.

A suscetibilidade de cães a este carrapato é discutível, pois, segundo Szabó; Bechara (1999), com base em análises histopatológicas de cães, não foram encontradas células que pudessem realizar uma efetiva defesa contra o parasito $R$. sanguineus, constatando que os canídeos não desenvolvem imunidade efetiva. No entanto, segundo Jittapalapong et al. (2000), nos Estados Unidos, existem diferentes graus de resistência para cães que sofreram reinfestaçóes por este ixodídeo, o que foi verificado no presente estudo, uma vez que animais residentes dos mesmos locais apresentaram graus de infestação totalmente distintos.

A massa das teleóginas pré e pós-desprendimento são similares àquelas apresentadas por SARTOR et al. (1996) e Bellato; Daemon (1997), os quais observaram uma massa média de 166,02 e 178,80 mg, respectivamente, para teleóginas desprendidas de coelhos. Cheuiche (1996) e Dantas-Torres et al. (2011) encontraram um total de 120,8 e 170,9 mg concomitantemente para fêmeas coletadas de seu hospedeiro preferencial. No entanto, BeCHARA et al. (1995), usando o cáo doméstico como animal experimental, em repetidas infestaçôes, constataram que teleóginas oriundas deste hospedeiro obtiveram uma massa média de $126 \mathrm{mg}$, inferior àquela verificada no presente estudo. Tais diferenças devem-se ao fato de as fêmeas coletadas para este estudo serem provenientes de infestaçóes naturais. Esses resultados sugerem que fêmeas de $R$. sanguineus podem responder de maneiras distintas quando submetidas ao estresse de uma colônia, mesmo tendo sido alimentadas em seu hospedeiro natural.

O período de pré-postura observado por CoelHo (1993) foi de quatro dias; Bechara et al. (1995) foi de 4,2 dias; Cheuiche (1996) encontrou 3,5 dias; e Bellato; Daemon (1997), 3,19 dias, o que é similar à média verificada neste estudo (quatro dias). Por outro lado, Dantas-Torres et al. (2011), na Itália, ao estudarem os efeitos do clima na biologia de $R$. sanguineus, adaptado às condiçóes de climas quente e seco, encontraram um período de pré-postura de 2,5 dias. Correlaçóes referentes à influência da água no ciclo de vida de $R$. sanguineus, realizadas por Yoder et al. (2006), demonstraram que este carrapato é extremamente adaptado a ambientes secos, o que facilitaria sua sobrevivência e o estabelecimento de populaçóes em condiçóes desfavoráveis de umidade. No entanto, Dantas-Torres et al. (2011) indicaram que altas temperaturas e baixa umidade são prejudiciais ao estabelecimento de populaçóes de $R$. sanguineus. Os resultados confirmam a importância da umidade relativa na bioecologia deste ixodídeo.

Bellato; Daemon (1997) encontraram uma média de massa dos ovos de 114,27 mg, enquanto Cheuiche (1996) e Dantas-Torres et al. (2011) notaram médias de 83,08 e $95 \mathrm{mg}$, respectivamente. Ambos os resultados vêm ao encontro dos verificados neste estudo $(100 \mathrm{mg})$, indicando que teleóginas adaptadas a climas diferentes, quando submetidas a condiçóes satisfatórias de umidade relativa, podem demonstrar uma oviposição similar.

Nos primeiros sete dias, foram ovipostos 99,0 e 98,0\% da massa total de ovos provenientes de fêmeas pré e pós-desprendimento, sendo que Bellato; Daemon (1997) avaliaram que, nas mesmas condiçōes, (temperatura $27 \pm$ $1^{\circ} \mathrm{C}$ e umidade relativa superior a $70,0 \%$ ) foram ovipostos 91,55\% da massa total de ovos. Coelho (1993) observou em infestações subsequentes que, nos sete primeiros dias, foram ovipostos $89,54 \%$ do total da massa de ovos na primeira geração, $81,33 \%$ para a segunda e $89,43 \%$ para a terceira. Possivelmente, essas diferenças devam-se ao hospedeiro utilizado nos estudos que, neste caso, foi o coelho. Fato que pode ser explicado devido a esse animal adquirir algum grau de imunidade frente às infestaçóes por $R$. sanguineus (Garin; Grabarev, 1972), além do fator de espécie específico. Dantas-Torres et al. (2011) constataram que fêmeas mais leves foram capazes de converter o sangue em ovos com maior rapidez, em comparaçáo àquelas com maior massa. Bellato; Daemon (1997) concluíram que a média da massa residual da fêmea (quenógina) foi de 35,6 mg, resultado semelhante ao constatado neste trabalho.

Quanto à eclodibilidade, Bellato; Daemon (1997) observaram um percentual médio de $84,43 \%$, em condiçôes similares de temperatura e umidade relativa utilizados no presente estudo. No entanto, ela foi obtida no presente trabalho com 94,0 e 95,0\%, respectivamente para teleóginas provenientes de diferentes origens. Essa diferença pode ser atribuída ao hospedeiro utilizado e também à avaliação subjetiva que é realizada para a análise desse parâmetro (Bellato; Daemon, 1997). Coelho (1993) e Bechara et al. (1995), ao estudarem carrapatos ingurgitados em cáes, observaram percentuais de eclodibilidade larval de 97,4 e 90,2\%, os quais se assemelham ao encontrado neste estudo.

Os resultados para longevidade larval são semelhantes aos encontrados para esse parâmetro por CHeuiche (1996) e Bellato; Daemon (1997).

Ao analisar os IER e IN, os dados registrados no estudo de 61,0 e $74,0 \%$, respectivamente, para fêmeas provenientes 
de ambas as origens, são correspondentes àqueles encontrados pelos demais autores. DANTAS-TorRes et al. (2011) verificaram um IER de 55,7\%, sendo que as diferenças encontradas podem estar associadas à capacidade individual que as fêmeas possuem de metabolizar o sangue em ovos.

Os parâmetros biológicos das populaçóes estudadas de R. sanguineus na regiáo Sul do Rio Grande do Sul, adaptadas às condiçóes de clima subtropical, são similares aos de populaçóes nativas de outras regióes com clima quente e tropical, como as do Rio de Janeiro (Bellato; Daemon, 1997) e de São Paulo, ambas no Brasil (Bechara et al., 1995). Tal fato permitirá que medidas de controle e intervalos de tratamentos recomendados nessas regióes possam ser utilizadas na área de estudo. No Rio Grande do Sul, grandes infestaçóes por $R$. sanguineus nas regiốes urbanas começaram a ser registradas somente há cerca de uma década (Ribeiro et al., 1997). Na região Sul do Estado, observou-se que o problema se agrava a cada ano que passa, exigindo medidas eficazes de controle.

A semelhança encontrada entre os parâmetros biológicos de carrapatos coletados pré e pós-desprendimento natural do hospedeiro pode vir a facilitar a obtenção de material para estudos epidemiológicos com esse ixodídeo, visto que são muito mais abundantes no ambiente. Isso ocorre devido à sua característica biológica de, ao final do repasto sanguíneo, desprender-se do hospedeiro quando este se encontra no interior da toca ou abrigo, a fim de garantir o ciclo nidícola naquele ambiente (Labruna; Pereira, 2001).

\section{CONCLUSÕES}

A coleta de teleóginas de $R$. sanguineus para estudos epidemiológicos pode ser realizada tanto do corpo do hospedeiro, antes de seu desprendimento natural, quanto do ambiente, antes de ser iniciada a postura, devido à similitude que ambos os grupos apresentaram em seus parâmetros biológicos. Além disso, concluiu-se que as populaçôes de $R$. sanguineus adaptadas ao clima subtropical da regiâo Sul do Rio Grande do Sul, Brasil, possuem parâmetros biológicos similares àquelas adaptadas a regióes mais quentes. Portanto, as medidas de controle adotadas nessas outras regióes do país podem ser recomendadas para esta do estudo.

| | | | | | | | | | | | | | | | | | | | | | | | | | | | | | | | | | | | | | | | | | | | | | | | | | | | | | | | | | | | | | | | | | | | | | | | | | | | | | | | | | | | | | | | | | | | | | | | | | | | | | | | | | | | | | | | | | | | | | | | | | | | | | | | | | | | | | | | | | | | | | | | | | | | | | | | | | | | | | | | | | | | | | | | | | | | | | | | | | | | | | | | | | | | | | | | | | | | | | | | | | | | | | | | | | | | |

\section{REFERÊNCIAS}

BECHARA, G.H.; SZABÓ, M.P.J.; FERREIRA, B.R.; GARCIA, M.V.Rhipicephalus sanguineus tick in Brazil: feeding and reproductive aspects under laboratorial conditions. Revista Brasileira de Parasitologia Veterinária, v.4, n.2, p.61-66, 1995.

BELLATO, V.; DAEMON, E. Efeito de três temperaturas sobre a fase não parasitária de Rhipicephalus sanguineus (Latreille, 1806) (Acari: Ixodidae). Revista Brasileira de Parasitologia Veterinária, v.6, n.1, p.21-27, 1997.

BENNET, G.F. Oviposition of Boophilus microplus (Canestrini) (Acarida: Ixodidae). I. Influence of tick size on egg production. Acarologia, v.16, n.1, p.52-61, 1974.

BURLINI, L.; TEIXEIRA, K.R.S.; SZABÓ, M.P.J.; FAMADAS, K.M. Molecular dissimilarities of Rhipicephalus sanguineus (Acari: Ixodidae) in Brazil and its relation with samples throughout the world: is there a geographical pattern? Experimental and Applied Acarology, v.50, n.4, p.361-374, 2010.

CHEUICHE, S.M.V.Ciclo de vida do Rhipicephalus sanguineus (Latreille, 1806) (Ixodidae) em condições laboratoriais em Porto Alegre, RS. 1996. 54p.Dissertação (Mestrado em Ciências Veterinárias) - Universidade Federal do Rio Grande do Sul, Rio Grande do Sul, 1996.

COELHO, C.F. Biologia da fase não parasitária de Rhipicephalus sanguineus (Latreille, 1806) (Acari: Ixodidae) sob condições de laboratório: aspectos da ovoposição. 1993. 52p.Dissertação
(Mestrado em Parasitologia Veterinária) - Universidade Federal do Rio de Janeiro, Rio de Janeiro, 1993.

CUNHA, N.C.; FONSECA, A.H.; REZENDE, J.; ROZENTAL, T.; FAVACHO, A.R.M.; BARREIRA, J.D.; MASSARDS, C.L.; LEMOS, E.R.S. First identification of natural infection of Rickettsia rickettsii in the Rhipicephalus sanguineus tick, in the State of Rio de Janeiro. Pesquisa Veterinária Brasileira, v.29, n.2, p.105-108, 2009.

DANTAS-TORRES, F.; FIGUEREDO, L.A.; DOMENICO, O. Seasonal variation in the effect of climate on the biology of Rhipicephalus sanguineus in southern Europe. Parasitology, v.138, p.527-536, 2011.

DANTAS-TORRES, F.; LARUSSO, V.; TESTINI, G.; PAIVACAVALCANTI, M. DE; FIGUEREDO, L.A.; STANNECK, D.; MENCKE, N.; BRANDÃO-FILHO, S.P.; ALVES, L.C.; OTRANTO, D. Detection of Leishmania infantum in Rhipicephalus sanguineus ticks from Brazil and Italy. Parasitology Research, v. 106, p.857-860, 2010.

GARIN, N.S.; GRABAREV, P.A. Immune reaction in rabbits and guinea pigs during repeated feeding on them of ixodid ticks Rhipicephalus sanguineus (Latr; 1806). Meditsinskaia Parazitologiia l, v.41, p.274-279, 1972.

GRAY, J.S.; DAUTEL, H.; ESTRADA-PEÑA, A.; KAHL, O.; LINDGREN, E. Effects of climate change on ticks and tick-borne diseases in Europe. Interdisciplinary Perspectives on Infectious Diseases, 2009, 12 p.doi:10.1155/2009/593232 
JITTAPALAPONG, S.; STICH, R.W.; GORDON, J.C.; WITTUM, T.E.; BARRIGA, O.O. Performance of female Rhipicephalus sanguineus (Acari: Ixodidae) fed on dogs exposed to multiple infestations or immunization with tick salivary gland or midgut tissues. Journal of Medical Entomology, v.37, n.4, p.601-611, 2000.

LABRUNA, M.B. Biologia-Ecologia de Rhipicephalus sanguineus (Acari: Ixodidae). Revista Brasileira de Parasitologia Veterinária, v.13, p.123-124, 2004.

LABRUNA, M.B; PEREIRA, M.C. Carrapatos em cães no Brasil. Clínica Veterinária, v.30, p.24-32, 2001.

LABRUNA, M.B.; OGRZEWALSKA, M.; MARTINS, T.F.; PINTER, A.; HORTA, M.C. Comparative susceptibilidade of larval stages of Amblyomma aureolatum, Amblyomma cajennense, and Rhipicephalus sanguineus to infection by Rickettsia rickettsii. Journal of Medical Entomology, v.45, p.1 156-1 159, 2008.

OLIVEIRA, P.R.; BECHARA, G.H.; DENARDI, S.E.; SAITO, K.C.; NUNES, E.T.; SZABÓ, M.P.J.; MATHIAS, M.I.C. Comparison of the external morphology of Rhipicephalus sanguineus (Latreille, 1806) (Acari: Ixodidae) ticks from Brazil and Argentina. Veterinary Parasitology, v.129, n.1-2, p.139-147, 2005.

PEGRAM, R.G.; KEIRANS, J.E.; CLIFFORD, C.M.; WALKER, J.B. Clarification of the Rhipicephalus sanguineus group (Acari, Ixodoidea, Ixodidae). II. R. sanguineus (Latreille, 1806) and related species. Systematic Parasitology, v.10, n.1, p.27-44, 1987.

RIBEIRO, V.L.S.; WEBER, M.A.; FETZER, L.O.; VARGAS, C.R.B. Espécies e prevalência das infestações por carrapatos em cães de rua da cidade de Porto Alegre, RS, Brasil. Ciência Rural, v.27, n.2, p.285-289, 1997.

SARTOR, A.A.; CUNHA, D.W.; DAEMON, E. Aspectos da biologia de Rhipicephalus sanguineus (Latreille, 1806) (Acari: Ixodidae) em condições de laboratório: fase parasitária de larvas, ninfas e fêmeas e não parasitária de larvas e ninfas. Revista Brasileira de Medicina Veterinária, v.18, n.1, p.14-17, 1996.

TALLAHASSEE. STATISTIX ${ }^{\circledR}$, Statistix 9.0. Analytical software. 1 ed. FL, USA, 2008.

SZABÓ, M.P.J.; BECHARA, G.H. Sequential histopathology at the Rhipicephalus sanguineus tick feeding site on dogs and guinea pigs. Experimental and Applied Acarology, v.23, n.11, p.915-928, 1999.

SZABÓ, M.P.J.; MANGOLD, A.J.; JOÃO, C.F.; BECHARA, G.H.; GUGLIELMONE, A.A. Biological and DNA evidence of two dissimilar populations of the Rhipicephalus sanguineus tick group (Acari: Ixodidae) in South America. Veterinary Parasitology, v.130, n.1-2, p.131-140, 2005.

WALKER, J.B.; KEIRANS, J.E.; HORAK, I.G. (ed.). The genus Rhipicephalus (Acari: Ixodidae): a guide to the brown ticks of the world. Cambridge University Press: Cambridge, 2000. 655p.

YODER, J.A.; BENOIT, J.B.; RELLINGER, E.J.; TANK, J.L. Developmental profiles in tick water balance with a focus on the new Rocky Mountain spotted fever vector, Rhipicephalus sanguineus. Medical and Veterinary Entomology, v.20, p.365-372, 2006. 